\title{
Breast Cancer Classification: A CAD System for a Combined Use of Elastography and B-Mode Sonography
}

Keywords: Breast cancer; Elastography imaging; Computer-Aided Diagnosis; Color map; Interobserver agreement

\begin{abstract}
Purpose: The aim of this study was to evaluate and compare the diagnostic performance of elastography, B-mode Ultrasound (US), and a combination of elastography and B-mode US for the differentiation between benign and malignant lesions.

Methods: A prospective study was carried out from July to December 2015, which 87 patients with 83 lesions were examined with conventional B-mode ultrasound and strain elastography. All the lesions had been proven by biopsy, resulting in 31 malignant and 52 benign lesions. A radiologist with 16 years of experience classified visually these cases. We also used a CAD sys-tem to classify the lesions classified visually by the most experienced radiologist and using a CAD system. The data obtained were compared with the results provided by another radiologist and a resident with 2 years of experience. Sensitivity, specificity and $A \cup C$ for the three observers using the $C A D$ system were calculated.
\end{abstract}

Results: The developed CADx system provided a diagnostic concordance in the classification of breast lesions from the different ways of contour determination (manual and automatic), allowing to reduce the diagnostic variability. In addition, the CADx system showed superior results to the visual analysis of the radiologist. When the radiologist associated both examinations (B-mode ultrasound and elastography), his visual analysis provided $87.1 \%, 55.8 \%$ and 0.714 of sensitivity, specificity and AUC, respectively. When we considered the result provided by the association between B-mode ultrasound and elastography images, the CADx system provided a comparative increase of about $7 \%$ of sensitivity and $17.2 \%$ of specificity, using the contour delimited by the most experienced radiologist. In addition, a positive influence was observed in the use of the computational tool by radiologists, since, on average, their sensitivity and specificity indexes also increased in relation to the conventional analysis, from $87.1 \%$ and $55.8 \%$ to $90.3 \%$ and $73.1 \%$, respectively.

Conclusion: Thus, it can be concluded that the developed CADX system performed well in distinguishing benign from malignant lesions for both B-mode ultrasound and elastography. The AUC obtained was higher than the radiologist's visual analysis in most of the cases analyzed.

\section{Introduction}

Breast Ultrasound (US) is an important complementary technique for screening and has been proven to be useful in differentiating benign from malignant masses, mainly in dense breasts [1]. The American College of Radiology developed the Breast Imaging Reporting and Data System (BI-RADS) ultrasound lexicon to provide a common language for reporting and to avoid ambiguity in the interpretation, improving the diagnostic efficiency of the ultrasound [2-4]. The sonographic characteristics are organized into five categories, which are: shape, orientation, margins, echo pattern and posterior acoustic

\section{Journal of}

\section{Cancer Sciences}

\author{
Marcomini KD ${ }^{1^{*}}$, Fleury EFC ${ }^{2}$ and H. Schiabel ${ }^{2}$ \\ ${ }^{\prime}$ Department of Electrical and Computer Engineering, University of São Paulo, \\ Brazil \\ ${ }^{I}$ Department of Electrical and Computer Engineering, University of So \\ Paulo,Brazil

\section{*Address for Correspondence} \\ Marcomini KD, Department of Electrical and Computer Engineering, \\ University of São Paulo, 400 Avenida Trabalhador São-Carlense, \\ 13566-590 São Carlos, SP, Brazil \\ Submission: 05 August, 2020 \\ Accepted: 12 September, 2020 \\ Published: 20 November, 2020 \\ Copyright: (๑) 2020 Marcomini KD, et al. This is an open access article \\ distributed under the Creative Commons Attribution License, which \\ permits unrestricted use, distribution, and reproduction in any medium, \\ provided the original work is properly cited.
}

transmission [5,6]. Unfortunately, its diagnostic specificity is poor and generate a significant number of false positive results, increasing biopsied cases [7].

Elastography has been introduced to overcome these limitations and obtain a more accurate characterization of breast lesions. This is a newly developed dynamic technique that uses ultrasound to provide an estimate of tissue stiffness by measuring the degree of distortion under the application of an external force. Like palpation during physical examination, elastography uses tissue deformation or strain, caused by compression and is estimated by precompression and post compression ultrasonic signals. Elastography has proven to be highly specific in the evaluation of lesions. However, strain elastography provided objective data on tissue stiffness via the quantity of tissue displacement $[4,7,8]$.

In this context, errors due to the subjectivity in boundaries definitions and superposition between benign and malignant characteristics are very common during the visual analysis of the specialist. With the advance of digital technology, mainly of the digital image processing - including pattern recognition and artificial intelligence - radiologists have the opportunity to improve the diagnostic accuracy with the aid of computer systems. Computer Aided-Diagnosis (CAD) is a technology which has been implemented with the purpose of providing double reading, working as a second opinion. CAD systems are useful when there is high interobserver variability, absence of trained observers or impossibility of performing double reading with two or more radiologists. Clinical studies have demonstrated that CAD increases sensitivity in the diagnostic of breast cancer [9-11].

This study presents the proposal of investigating the diagnostic ability of a computational system in the characterization of suspicious findings in B-mode ultrasound and breast elastography imaging. The system provides the individual result of each exam, as well as the result of the combination of them, proving to be an innovative classification proposal. We also evaluated the performance of this system in the combined diagnostic with the specialist.

\section{Materials and Methods}


Citation: Marcomini KD, Fleury EFC, Schiabela H. Breast Cancer Classification: A CAD System for a Combined Use of Elastography and B-Mode Sonography. J Cancer Sci. 2020;7(1): 6.

\section{ISSN: 2377-9292}

\section{Image database}

The local institutional review board approved this study (Protocol No. 53543016.2.0000.0072) and the consent was obtained from all patients. A radiologist with 2 years of experience performed the B-mode ultrasound and Strain Elastography (SE) examinations using a Toshiba Aplio 400 Ultrasound System (Toshiba, Japan) with a 5-10 $\mathrm{MHz}$ linear transducer.

The target population was comprised of 83 consecutive female patients, represented by 92 solid lesions. However, we excluded five patients because they presented non-mass lesions on the ultrasound before the percutaneous biopsy confirmation. A total of 83 lesions were included in this study, resulting in 31 malignant and 52 benign lesions. All lesions underwent excisional biopsy, core needle biopsy or fine-needle aspiration biopsy for pathologic diagnosis, used as the gold standard for evaluation of the CAD. The collection of cases was from July to December 2015 during diagnostic breast exams at the Brazilian Institute for Cancer Control (IBCC - São Paulo, SP, Brazil).

The images were deidentified for patient confidentiality. The SE image was superimposed on the corresponding B-mode grayscale image with a color scale. In the color scale, blue indicates soft tissue and red indicates hard tissue. B-mode images were on the right side and elastographic images were on the left side.

\section{Delimitation of the lesion}

We evaluated the diagnosis from manual delimitation and using an automatic segmentation technique.

Three radiologists draw the contour on the B-mode ultrasound images. The first has 16 years of experience in breast imaging, the second has 10 years and the last one was a second-year resident.

In the automatic segmentation, we used the active contour technique based on the Mumford-Shah and level set functions [12]. We also applied a post-processing to remove disconnected pixels and join internal valleys, as described in a previous work [13].

\section{Classification in B-mode ultrasound imaging}

The quantitative features extracted from segmented image can be classified, according to the fifth edition of Breast Imaging Reporting

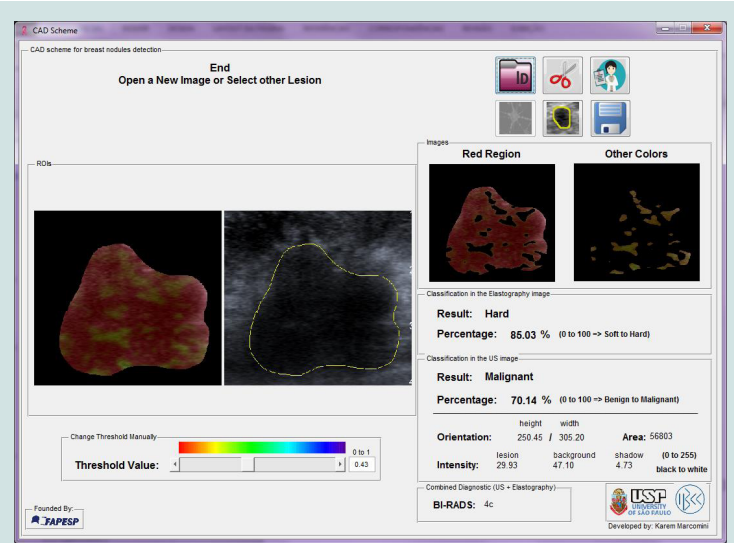

Figure 1: Representative image of setting the region of interest for CAD system analysis. The margin of the breast mass was defined manually for analysis (yellow line). The ultrasonographic and elastographic features were automatically analyzed by the CAD system, and a final assessment was visualized.

and Data System (BI-RADS), into five categories: shape, orientation, margin, echo pattern and posterior acoustic features. Ten features were de-fined by in order to quantify these BI-RADS features, as presented in [14] Table 1.

In order to distinguish benign from malignant lesions, we used the Support Vector Machine (SVM) [16], which is a technique that seeks an optimal hyperplane to separate two classes of samples. The function FITCSVM was applied to create the routine in MATLAB. 10 -fold cross-validation was used to evaluate the performance of the classifier. The best performance of the classifier was using the features ADEE, orientation, NumPeaks, entropy and lesion size. The procedure applied to select features and classify the lesions were described in more detail by [17].

\section{Classification in elastography imaging}

In order to measure the amount of hard tissue (i.e., tissues in red) in the lesion, we developed an algorithm to segment red areas and quantify its predominance within the lesion, allowing us to classify it as soft, intermediate, or hard. This algorithm converts the image from RGB to CIELab color space and, after that, we applied Otsu method

Table 1: Summary of the features that quantify each BI-RADS category.

\begin{tabular}{|c|c|}
\hline $\begin{array}{l}\text { BI-RADS } \\
\text { category }\end{array}$ & Description \\
\hline Shape & $\begin{array}{c}\text { Area difference with equivalent ellipse (ADEE) describes how irregular the tumor is. It is calculated by the difference between the tumor and its } \\
\text { equivalent ellipse. }\end{array}$ \\
\hline Orientation & Describes the direction of long axis of the tumor. If the long axis is parallels or anti-parallels the skin. \\
\hline \multirow{4}{*}{ Margin } & Average of vector difference (AvgDiff): Average intensity difference between inside and outside contours. \\
\hline & Number of peaks on the distance vector (Num Peaks) is the number of local maxima of vector difference. \\
\hline & $\begin{array}{l}\text { Average of the distance vector (Avg Distance): A convex hull of the tumor is draw, and a distance vector between the tumor contour and its convex } \\
\text { hull is computed. }\end{array}$ \\
\hline & $\begin{array}{l}\text { Area difference between the convex hull and tumor }(\mathrm{ADCH}) \text { can depict the irregularity of the tumor margin. It is the ratio between de difference of the } \\
\text { number of pixels within the convex and the tumor by the number of pixels within the tumor. }\end{array}$ \\
\hline \multirow{2}{*}{ Echo pattern } & Echogenicity is the intensity difference between the surrounding area and the tumor area. \\
\hline & Entropy describes the degree of heterogeneity in the tumor. \\
\hline $\begin{array}{l}\text { Posterior } \\
\text { feature }\end{array}$ & Shadow: The average intensity a rectangular region below the tumor is compared with the average intensity of the tumor. \\
\hline-- & $\begin{array}{c}\text { Lesion size is not a BI-RADS feature. However, the fifth edition of BI-RADS Ultrasound [15] does mention that lesion size should be given to report } \\
\text { important findings and might be combined with other features to improve the performance of tumor classifiers. }\end{array}$ \\
\hline
\end{tabular}


Citation: Marcomini KD, Fleury EFC, Schiabela H. Breast Cancer Classification: A CAD System for a Combined Use of Elastography and B-Mode Sonography. J Cancer Sci. 2020;7(1): 6.

ISSN: 2377-9292

Table 2: Classification of the breast lesions after the addition of the elastography results (soft, intermediate and hard) to the BI-RADS lexicon [21].

\begin{tabular}{|c|c|c|c|c|}
\hline \multicolumn{2}{|c|}{} & \multicolumn{3}{c|}{ Elastography } \\
\cline { 2 - 5 } \multicolumn{1}{|c|}{} & Soft & Intermediate & Hard \\
\hline \multirow{4}{*}{ B-Mode Ultrasound } & BI-RADS 3 & BI-RADS 2 & BI-RADS 3 & BI-RADS 4a \\
\cline { 2 - 6 } & BI-RADS 4a & BI-RADS 3 & BI-RADS 4a & BI-RADS 4b \\
\cline { 2 - 6 } & BI-RADS 4b & BI-RADS 4a & BI-RADS 4b & BI-RADS 4c \\
\cline { 2 - 6 } & BI-RADS 4c & BI-RADS 4b & BI-RADS 4c & BI-RADS 5 \\
\cline { 2 - 6 } & BI-RADS 5 & BI-RADS 4c & BI-RADS 5 & BI-RADS 5 \\
\hline
\end{tabular}

Table 3: Classification of breast lesions in ultrasonography.

\begin{tabular}{|c|c|c|c|}
\hline Classification & Sensitivity & Specificity & AUC \\
\hline $\begin{array}{c}\text { Manual delineation } \\
\text { (Radiologist 1) + CAD }\end{array}$ & $87.10 \%$ & $71.15 \%$ & 0.843 \\
\hline $\begin{array}{c}\text { Manual delineation } \\
\text { (Radiologist 2) + CAD }\end{array}$ & $90.32 \%$ & $59.62 \%$ & 0.800 \\
\hline $\begin{array}{c}\text { Manual delineation } \\
\text { (Resident) + CAD }\end{array}$ & $80.65 \%$ & $46.15 \%$ & 0.798 \\
\hline Automatic Segmentation + CAD & $61.29 \%$ & $80.77 \%$ & 0.798 \\
\hline $\begin{array}{c}\text { Visual Analysis - } \\
\text { Radiologist 1 }\end{array}$ & $96.77 \%$ & $57.69 \%$ & 0.723 \\
\hline
\end{tabular}

on the $\mathrm{a}^{*}$ channel $[18,19]$.

We classified the lesions in elastography images into three categories: (1) soft when the red area is lower than $50 \%$ of the total lesion area; (2) intermediate when this value is between $50-75 \%$; and (3) hard when the red area is larger than $75 \%$ of the total area. We considered the lesions classified as soft and intermediate as negative and hard as positive cases. We included in the system the possibility of the specialist to change the threshold value. This value defines whether the pixel is red or any other color. The specialist can change the threshold value if he considers that the color distribution is not entirely accurate based on his visual perception [20].

\section{Association between ultrasound and elastography}

Fleury (2015) proposed an association criterion between ultrasound and elastography results in order to provide a single diagnosis. This criterion is presented in Table 2.

To use this classification criterion, we assign percentage values for the tendency to malignity or benignity, since the SVM provides a binary result, where zero defines the lesion as benign and 1 as malignant.

To make it possible to use this classification criterion, we used the values of separation between the hyperplanes. The value of separation ranges between -1 and +1 . Thus, the values between +1 and -1 represents the variation from zero to $100 \%$, where zero is for benign and $100 \%$ for malignant lesion.

We defined thresholds to represent each BI-RADS category, which are:

-BI-RADS 3: malignancy percentage less than 50\%.

BI-RADS 4a: malignancy percentage between 50 and 64\%.

- BI-RADS 4b: malignancy percentage between $64 \%$ and $77 \%$.

- BI-RADS 4c: malignancy percentage between $77 \%$ and $90 \%$.

- BI-RADS 5: malignancy percentage greater than 90\%.

\section{Image review and application of the CAD system}

A radiologist with 16 years of breast imaging experience reviewed the data for analysis. The observer was blinded to clinical information and pathologic results of each mass during image review. After image review by the radiologist, the CAD system was applied to the same image the radiologist used for his analysis. The Region of Interest (ROI) was either automatically or manually drawn along the border of the mass by CAD system. The results of the CAD and final assessments were immediately displayed and recorded for data analysis Figure 1. After being informed of the final assessment made by CAD system, the radiologist gave a final assessment for each breast mass, integrating the results of the CAD system.

\section{Data evaluation and statistical analysis}

Final assessment based on BI-RADS criterion were also divided into 2 groups for statistical analysis: positive assessments consisted of categories 4a to 5, and negative assessments consisted of categories 2 and 3. Diagnostic performance of the radiologist, CAD system and the integration of CAD with the radiologist were analyzed and compared, including sensitivity, specificity, and area under the receiver operating characteristic curve (AUC).

\section{Results}

\section{Classification of B-mode ultrasound}

We applied the classifier developed by on our image database and the results are presented in [17] Table 3.

Data from visual analysis are related to the clinical diagnosis provided by Radiologist 1 not using the computational tool to assist in his diagnosis. However, Radiologist 1 was the only one who performed the visual analysis.

\section{Classification of elastography}

For this stage, we performed two experiments. Experiment 1: we investigated the accuracy of the fully automatic classifier, i.e., we quantified the pixels representing the hard tissue by using Otsu method, from the $\mathrm{a}^{*}$ channel. In Experiment 2, the specialist could change the threshold value to adjust the color distribution, this procedure can include or remove the amount of tissue defined as red by the automatic threshold Table 4 .

\section{Combined diagnostic of elastography and B-mode ultrasound}

For the final diagnosis, we associated the results of both examinations according to the criterion presented in item 2.E

Table 5 shows the sensitivity, specificity and AUC values for the diagnostic combination of the CAD system for B-mode ultrasound and elastography. We evaluated the result from elastography CAD system with and without the radiologist intervention.

\section{Final assessment between the radiologist and the CAD system}

Interobserver variability is inevitable, and it can lead to inconsistent diagnoses among radiologists [22,23]. CAD systems have recently been used to overcome this variability and increase the diagnostic accuracy of breast lesions. 
Citation: Marcomini KD, Fleury EFC, Schiabela H. Breast Cancer Classification: A CAD System for a Combined Use of Elastography and B-Mode Sonography. J Cancer Sci. 2020;7(1): 6.

ISSN: $2377-9292$

Table 4: Classification of breast lesions in elastography.

\begin{tabular}{|c|c|c|c|c|}
\hline Experiment & Classification & Sensitivity & Specificity & AUC \\
\hline \multirow{4}{*}{ I } & Manual delineation (Radiologist 1) + CAD & $70.97 \%$ & $88.46 \%$ & 0.853 \\
\hline & Manual delineation (Radiologist 2) + CAD & $67.70 \%$ & $84.60 \%$ & 0.806 \\
\hline & Manual delineation (Resident) + CAD & $51.60 \%$ & $92.30 \%$ & 0.814 \\
\hline & Automatic segmentation + CAD & $67.74 \%$ & $80.77 \%$ & 0.743 \\
\hline \multirow{4}{*}{ II } & Manual delineation (Radiologist 1) + CAD & $80.65 \%$ & $88.46 \%$ & 0.883 \\
\hline & Manual delineation (Radiologist 2) + CAD & $80.65 \%$ & $88.46 \%$ & 0.892 \\
\hline & Manual delineation (Resident) + CAD & $80.65 \%$ & $73.08 \%$ & 0.811 \\
\hline & Visual Analysis - Radiologist 1 & $61.29 \%$ & $88.46 \%$ & 0.829 \\
\hline
\end{tabular}

Table 5: Result of the diagnostic combination between B-mode ultrasound and elastography.

\begin{tabular}{|c|c|c|c|c|}
\hline Experiment & Classification & Sensitivity & Specificity & AUC \\
\hline \multirow{4}{*}{ I } & Manual delineation (Radiologist 1) + CAD & $93.55 \%$ & $65.38 \%$ & 0.795 \\
\hline & Manual delineation (Radiologist 2) + CAD & $100.00 \%$ & $59.62 \%$ & 0.798 \\
\hline & Manual delineation (Resident) + CAD & $93.55 \%$ & $51.92 \%$ & 0.727 \\
\hline & Automatic segmentation + CAD & $80.65 \%$ & $75.00 \%$ & 0.778 \\
\hline \multirow{4}{*}{ II } & Manual delineation (Radiologist 1) + CAD & $93.55 \%$ & $65.38 \%$ & 0.795 \\
\hline & Manual delineation (Radiologist 2) + CAD & $100.00 \%$ & $61.54 \%$ & 0.808 \\
\hline & Manual delineation (Resident) + CAD & $96.77 \%$ & $38.46 \%$ & 0.676 \\
\hline & Visual Analysis - Radiologist 1 & $97.77 \%$ & $59.62 \%$ & 0.782 \\
\hline
\end{tabular}

Table 6: CAD influence on the radiologist's classification.

\begin{tabular}{|c|c|c|}
\hline Classification & Sensitivity & Specificity \\
\hline Visual Analysis - Radiologist 1 & $87.10 \%$ & $55.77 \%$ \\
\hline Visual Analysis (Radiologist 1) after the CAD system information & 0.714 & $90.32 \%$ \\
\hline
\end{tabular}

The final evaluation consisted of the integration of radiologist and CAD system. For this analysis, B-mode ultrasound and elastography image were presented to the radiologist, who provided the diagnosis based on his visual analysis. Then, the results of the system (individual and with the association of the exams) were shown to him. The radiologist classified the lesion again and we can evaluated the influence of CAD on his diagnosis. The results obtained are shown in Table 6.

\section{Discussion}

The analysis of the images, in general, occurs through the visual analysis performed by one, or when possible, more specialists. However, such visual analysis may result in significant inter and intraobserver variability, even when the procedure is performed under the same conditions $[24,25]$. Some studies report the interobserver variability in the diagnosis of lesions in breast ultrasound and elastography imaging [26-29].

Computer-aided diagnosis systems were recently applied to overcome the variability observer as well as to improve the diagnostic performances. Our CAD system applies a novel classification technique, providing diagnoses for breast lesions found on B-mode ultrasound and elastography images. In addition, it is possible to obtain the combined diagnosis of the exams (US + elastography).

Among the methods of machine learning, SVM is a classifier that has been widely used to distinguish benign from malignant lesions in B-mode ultrasound imaging [14,30], including commercial systems such as, for example, S-detect and B-CAD [23,31,32]. Our study showed a superior result to S-detect - whose AUC was 0.815 , while the proposed CAD system was 0.843 , considering the manual delineation of the most experienced radiologist.
Elastography provides significant information regarding tissue elasticity and suspicious findings. This information is expressed by color variation during compression and after decompression of ROI. The proposed approach is simple and capable of increasing diagnostic specificity, as presented in some studies and comparable or superior to the results of other systems [33-36]. The system allows parameter adjustment in order to increase the diagnostic accuracy as reported in Experiment 2, where Radiologist 2 was able to improve diagnostic accuracy, obtaining AUC value greater than that Radiologist 1 at $1.02 \%$.

The model developed for classification in elastography images is related to the shape of the lesion (segmented area), the AUC value for the automatic method is lower than the value obtained by radiologists due to the inclusion of surrounding tissues. However, when the result is associated with that obtained in the classification of the B-mode ultrasound image, the AUC value approximates that provided with the manual contour delimitation of Radiologists 1 and 2 and is higher than that of the Resident.

From the data shown in Table 5, we observed that the interference of Radiologist 1 in the threshold value was not significant when this classification is associated with that of the B-mode ultrasound, since the sensitivity, specificity and AUC were not changed. When Radiologist 2 changed threshold value, specificity and AUC increased by $3.22 \%$ and $1.25 \%$, respectively. On the other hand, the value of specificity and AUC decreased when the Resident changed the value of the threshold.

We did not include the automatic segmentation in Experiment 2 due to the absence of an observer to perform the change in threshold value. However, the user can change the threshold value if he thinks it is necessary. It is important to highlight the change of this value can 
Citation: Marcomini KD, Fleury EFC, Schiabela H. Breast Cancer Classification: A CAD System for a Combined Use of Elastography and B-Mode Sonography. J Cancer Sci. 2020;7(1): 6.

modify the result of the classification in the elastography and also in the combined diagnosis.

The visual evaluation of the radiologist with the aid of the CAD system provided a substantial increase in the specificity rate from $55.77 \%(29 / 52)$ to $73.08 \%$ (38/52) - addition of 11 true Negative cases (VN) and two False Positives (FP). In relation to sensitivity, the variation was lower, about $3 \%$ - increase of two true Positive cases (PV) and one false Negative (VN), resulting in a sensitivity of $90.32 \%$ (28/31). Thus, we can affirm that the developed system was able to improve overall diagnosis, increasing the sensitivity and specificity when used in conjunction with the specialist's clinical evaluation, providing a significant increase in AUC value (14.43\%).

The main reasons for the radiologist's change of opinion after he sees the result of the CAD sys-tem are related to the visualization of the morphology of the lesion after the manual delineation and the distribution/quantification of the hard and soft tissues within the lesion in elastography imaging. This factor may be related to the more precise visualization of the margins and the shape of the lesion when it is seen with the overlap of the contour.

In addition, the behavior of the CAD system was similar to the visual analysis of the radiologist, reaching a higher sensitivity rate in the diagnosis of lesions seen in the B-mode ultrasound and in the association of the exams and, for the elastography, a higher specificity rate.

In conclusion, our initial experience with ultrasound breast elastography showed that it was more specific and more accurate than conventional ultrasound. Combining our system with the experience of the radiologist can improve the specificity and can potentially reduce unnecessary breast biopsies.

\section{References}

1. Youk JH, Son EJ, Gweon HM, Kim H, Park YJ, et al. (2014) Comparison of strain and shear wave elastography for the differentiation of benign from malignant breast lesions, combined with B-mode ultrasonography: qualitative and quantitative assessments. Ultrasound Med Biol 40: 2336-2344.

2. D'Orsi C, Sickles E, Mendelson E, Morris E (2013) Breast imaging reporting and data system: ACR BI-RADS-breast imaging atlas. ( $5^{\text {th }}$ edn). Reston, VA. American College of Radology.

3. Hao SY, Jiang QC, Zhong WJ, Zhao XB, Yao JY, et al. (2016) Ultrasound elastography combined with BI-RADS-US classification system: is it helpful for the diagnostic performance of conventional Ultrasonography? Clin Breast Cancer 16: e33-e41.

4. Arslan S, Uslu N, Ozturk FU, Akcay EY, Tezcaner T, et al. (2017) Can strain elastography combined with ultrasound breast imaging reporting and data system be a more effective method in the differentiation of benign and malignant breast lesions? J Med Ultrason 44: 289-296.

5. Hong AS, Rosen EL, Soo MS, Baker JA (2005) BI-RADS for sonography: positive and negative predictive values of sonographic features. AJR Am J Roentgenol 184: 1260-1265.

6. Kim SY, Park JS, Koo HR (2015) Combined use of ultrasound elastography and B-Mode sonography for differentiation of benign and malignant circumscribed breast masses. J Ultrasound Med 34: 1951-1959.

7. Goddi A, Bonardi M, Alessi S (2012) Breast elastography: A literature review. J Ultrasound 15: 192-198.

8. Mutala TM, Ndaiga P, Aywak A (2016) Comparison of qualitative and semiquantitative strain elastography in breast lesions for diagnostic accuracy. Cancer Imaging 16: 12
9. Stavros AT (2009) New advances in breast ultrasound: computer-Aided detection. Ultrasound Clin 4: 285-290.

10. Drukker K, Pesce LL, Giger ML (2010) Repeatability and classifier bias in computer-aided diagnosis for breast ultrasound. In: Karssemeijer N, Summers RM (eds). Proc SPIE, Medical Imaging: Computer-Aided Diagnosis pp. 76242B.

11. Yap MH, Edirisinghe EA, Bez HE (2004) Object boundary detection in ultrasound images. $3^{\text {rd }}$ Can. Conf Comput Robot Vis., IEEE pp. 1-6.

12. Chan TF, Vese LA (2001) Active contours without edges. IEEE Trans Image Process 10: 266-277.

13. Marcomini KD, Fleury EFC, Oliveira VM, Carneiro AAO, Nishikawa RM, et al. (2016) Classification of lesions in breast elastography images based on strain: A performance evaluation between manual and automatic segmentation. CBEB, XXV Congresso Brasileiro de Engenharia Biomédica pp. 1135-1138.

14. Shan J, Alam SK, Garra B, Zhang Y, Ahmed T (2015) Computer-aided diagnosis for breast ultrasound using computerized BI-RADS features and machine learning methods. Ultrasound Med Biol 42: 980-988.

15. Mendelson EB, Böhm-Vélez M, Berg WA, Whitman GJ, Feldman MI, et al (2013) ACR BI-RADS ${ }^{\circledR}$ Ultrasound. ACR BI-RADS ${ }^{\circledR}$ Atlas, Breast Imaging Report Data Syst Reston. VA Am Coll Radiol 135-131.

16. Vapnik VN (1998) Statistical learning theory 1: 768.

17. Fleury E, Marcomini K (2019) Performance of machine learning software to classify breast lesions using BI-RADS radiomic features on ultrasound images. Eur Radiol Exp 3: 34

18. Otsu N (1979) A threshold selection method from gray-level histograms. IEEE Trans Syst Man Cybern ML 9: 62-66.

19. Marcomini KD, Fleury EFC, Oliveira VM, Carneiro AAO, Schiabel H, et al. (2018) Evaluation of a computer-aided diagnosis system in the classification of lesions in breast strain elastography imaging. Bioengineering 5: 2-14.

20. Marcomini KD, Fleury EFC, Oliveira VM, Carneiro AAO, Schiabel $\mathrm{H}$, et al (2017) Agreement between a computer-assisted tool and radiologists to classify lesions in breast elastography images. In: Armato SG, Petrick NA (eds). Proc SPIE, Medical Imaging: Computer-Aided Diagnosis 10134: $101342 \mathrm{~T}$.

21. Fleury EFC (2015) The importance of breast elastography added to the BIRADS(R) ( $5^{\text {th }}$ edn) lexicon classification. Rev Assoc Med Bras 61: 313-316.

22. Horsch K, Giger ML, Vyborny CJ, Venta LA (2004) Performance of computeraided diagnosis in the interpretation of lesions on breast sonography. Acad Radiol 11: 272-280.

23. Cho E, Kim E, Song MK, Yoon JH (2017) Application of Computer-Aided Diagnosis on Breast Ultrasonography: Evaluation of Diagnostic Performances and Agreement of Radiologists According to Different Levels of Experience. J Ultrasound Med 37: 209-216.

24. Zhang X, Xiao Y, Zeng J, Qiu W, Qian M, et al. (2014) Computer-assisted assessment of ultrasound real-time elastography: Initial experience in 145 breast lesions. Eur J Radiol 83: e1-e7.

25. Zhi H, Xiao XY, Yang HY, Wen YL, Ou B, et al. (2008) Semi-quantitating stiffness of breast solid lesions in ultrasonic elastography. Acad Radiol 15 1347-1353

26. Calas M, Almeida R, Gutfilen B, Pereira W (2012) Interobserver concordance in the BI-RADS classification of breast ultrasound exams. Clinics 67: 185189.

27. Elverici E, Zengin B, Nurdan Barca A, Didem Yilmaz P, Alimli A, et al. (2013) Interobserver and intraobserver agreement of sonographic birads lexicon in the assessment of breast masses. Iran J Radiol 10: 122-127.

28. Lee HJ, Kim EK, Kim MJ, Youk JH, Lee JY, et al. (2008) Observer variability of Breast Imaging Reporting and Data System (BI-RADS) for breast ultrasound. Eur J Radiol 65: 293-298.

29. Yoon JH, Kim MH, Kim EK, Moon HJ, Kwak JY, et al. (2011) Interobserve variability of ultrasound elastography: how it affects the diagnosis of Breast lesions. AJR Am J Roentgenol 196: 730-736. 
Citation: Marcomini KD, Fleury EFC, Schiabela H. Breast Cancer Classification: A CAD System for a Combined Use of Elastography and B-Mode Sonography. J Cancer Sci. 2020;7(1): 6.

ISSN: 2377-9292

30. Huang YL, Chen DR, Jiang YR, Kuo SJ, Wu HK, et al. (2008) Computeraided diagnosis using morphological features for classifying breast lesions on ultrasound. Ultrasound Obstet Gynecol 32: 565-572.

31. Kim K, Song MK, Kim E, Yoon JH (2017) Clinical application of S-Detect to breast masses on ultrasonography: a study evaluating the diagnostic performance and agreement with a dedicated breast radiologist. Ultrasonography 36: 3-9.

32. Chabi ML, Borget I, Ardiles R, Aboud G, Boussouar S, et al. (2012) Evaluation of the accuracy of a Computer-aided Diagnosis (CAD) system in breast ultrasound according to the Radiologist's Experience. Acad Radiol 19: 311 319.
33. Lee SH, Chang JM, Cho N, Koo HR, Yi A, Kim SJ, et al. (2014) Practice guideline for the performance of breast ultrasound elastography. Ultrasound 33: 3-10.

34. Berg WA, Cosgrove DO, Doré CJ, Schäfer FKW, Svensson WE, et al. (2012) Shear-wave elastography improves the specificity of Breast US: the BE1 multinational study of 939 masses. Radiology 262: 435-449.

35. Athanasiou A, Tardivon A, Tanter M, Sigal-Zafrani B, Bercoff J, et al. (2010) Breast lesions: quantitative elastography with supersonic shear imaging-preliminary results. Radiology 256: 297-303.

36. Chang RF, Shen W, Yang MC, Moon WK, Takada E, et al. (2008) Computeraided diagnosis of breast color elastography. In: Giger ML, Karssemeijer N, (eds). Proc SPIE. Medical Imaging: Computer-Aided Diagnosis 6915: 69150I.

\section{Acknowledgement}

This work was supported by the São Paulo Research Foundation (FAPESP) grant \#2012/24006-5. The content is solely the responsibility of the authors and does not necessarily represent the official views of FAPESP. 